\title{
Cognitive Impairment in Mild Traumatic Brain Injury: A Longitudinal Diffusional Kurtosis and Perfusion Imaging Study
}

\author{
E.J. Grossman, J.H. Jensen, J.S. Babb, Q. Chen, A. Tabesh, E. Fieremans, D. Xia, M. Inglese, and R.I. Grossman
}

\begin{abstract}
BACKGROUND AND PURPOSE: Cognitive impairment is frequent among patients with mild traumatic brain injury despite the absence of detectable damage on conventional MR imaging. In this study, the quantitative MR imaging techniques DTI, DKI, and ASL were used to measure changes in the structure and function in the thalamus and WM of patients with MTBI during a short follow-up period, to determine whether these techniques can be used to investigate relationships with cognitive performance and to predict outcome.
\end{abstract}

MATERIALS AND METHODS: Twenty patients with MTBI and 16 controls underwent MR imaging at 3T and a neuropsychological battery designed to yield measures for attention, concentration, executive functioning, memory, learning, and information processing. MK, FA, $M D$, and CBF were measured in the thalamus by using region-of-interest analysis and in WM by using tract-based spatial statistics. Analyses were performed comparing regional imaging measures of subject groups and the results of testing of their associations with neuropsychological performance.

RESULTS: Patients with MTBI exhibited significant differences from controls for DTI, DKI, and ASL measures in the thalamus and various WM regions both within 1 month after injury and $>9$ months after injury. At baseline, DTI and DKI measures in the thalamus and various WM regions were significantly associated with performance in different neuropsychological domains, and cognitive impairment was significantly associated with MK in the thalamus and FA in optic radiations.

CONCLUSIONS: Combined application of DTI, DKI, and ASL to study MTBI might be useful for investigating dynamic changes in the thalamus and WM as well as cognitive impairment during a short follow-up period, though the small number of patients examined did not predict outcome.

ABBREVIATIONS: $\mathrm{ASL}=$ arterial spin-labeling; $\mathrm{DKI}=$ diffusional kurtosis imaging; $\mathrm{FA}=$ fractional anisotropy; $\mathrm{MD}=$ mean diffusivity; $\mathrm{MK}=$ mean $\mathrm{kurtosis} ; \mathrm{MTBI}=$ mild traumatic brain injury

M ild traumatic brain injury is one of the most significant public health problems in the United States. Each year approximately 1.1 million cases are reported, of which $\geq 30 \%$ expe-

Received June 25, 2012; accepted after revision August 14.

From the Department of Radiology (E.J.G., J.S.B., Q.C., E.F., D.X., M.I., R.I.G.), Center for Biomedical Imaging, and Department of Physiology and Neuroscience (E.J.G.), New York University School of Medicine, New York, New York; Department of Radiology and Radiological Science (J.H.J., A.T.), Medical University of South Carolina, Charleston, South Carolina; and Department of Neurology (M.I.), Mount Sinai School of Medicine, New York, New York.

This work was supported by the National Institutes of Health grants R01NS039135 and R01NS051623.

Please address correspondence to Elan Grossman, PhD, Department of Radiology, Center for Biomedical Imaging, New York University School of Medicine, 660 First Ave, 4th Floor, Room 420, New York, New York 10016; e-mail:

Elan.Grossman@nyumc.org

http://dx.doi.org/10.3174/ajnr.A3358

- Indicates open access to non-subscribers at www.ajnr.org

三 Indicates article with supplemental on-line table

Indicates article with supplemental on-line figures rience long-term cognitive impairment and $\geq 20 \%$ are unable to resume work, resulting in $>\$ 17$ billion in economic cost. ${ }^{1}$ Early medical and rehabilitative intervention may reduce chronic sequelae, but it is difficult to identify high-risk patients since diagnostic testing is suboptimal for predicting outcome ${ }^{2}$ and both CT and conventional MR imaging usually fail to detect any evidence of brain damage. ${ }^{3}$ Nevertheless, most pathologic models of MTBI assume that diffuse axonal injury in WM is present but cannot be resolved by using standard clinical imaging. ${ }^{3}$ In addition, it has been suggested that the thalamus may be an important further site of injury, though its role has remained largely underinvestigated. ${ }^{4-15}$

Recent MR imaging studies of MTBI have demonstrated that DTI can detect microscopic tissue damage, grade its severity, and provide markers that may be capable of predicting outcome. ${ }^{2,7,9,10,16,17}$ More recently, our group has used DTI in combination with $\mathrm{DKI}^{18-20}$ in a cross-sectional study of patients with MTBI. ${ }^{7}$ Unlike DTI, which assumes that water diffusion has a 
Gaussian displacement probability distribution and is useful in assessing the magnitude of diffusion, DKI measures how much the diffusion displacement probability distribution departs from a Gaussian form and is useful in assessing intravoxel diffusional heterogeneity, which is an indicator of complexity. Results demonstrated that DTI and DKI provided different and complementary information to supply a more sensitive and specific marker for tissue damage, which might also be capable of predicting outcome.

Some recent studies conducting functional investigations of MTBI have also reported impairment of perfusion and have demonstrated that changes in CBF might play a role in the development of posttraumatic morbidity and may provide prognostic makers for clinical outcome. ${ }^{4,6,12-14}$ In a previous cross-sectional study, ${ }^{6}$ we applied segmented true fast imaging with steadystate precession $\mathrm{ASL}^{21}$ to the investigation of patients with MTBI, a newly developed MR imaging technique that uses blood water as an endogenous freely diffusible tracer that can provide $\mathrm{CBF}$ measurements in the thalamus and deep gray matter while excluding susceptibility artifacts commonly associated with these regions in other similar approaches to data acquisition. Results demonstrated that in patients with MTBI, CBF in the thalamus was decreased and was associated with neuropsychological performance.

The aims of this study were the following: 1) to investigate whether DTI, DKI, and ASL can detect disease-related tissue changes during a short follow-up period in the thalamus, deep gray matter, and WM of patients with MTBI; 2) to investigate the dynamics of structural and functional changes triggered by brain injury in the thalamus, deep gray matter, and WM of patients with MTBI; and 3) to investigate whether DTI, DKI, and ASL baseline measures can predict cognitive outcome in patients with MTBI.

\section{MATERIALS AND METHODS Subjects}

Twenty consecutive patients with MTBI (16 men, 4 women; mean age, $34.8 \pm 10.7$ years; mean formal education, $15.2 \pm$ 1.87 years) were prospectively recruited for a baseline visit within 1 month after injury and were classified with MTBI by using diagnostic criteria developed by the Mild Traumatic Brain Injury Interdisciplinary Special Interest Group of the American Congress of Rehabilitation Medicine. ${ }^{22,23}$ Enrollment was permitted only in cases in which there were no other history of brain damage or disorders of the central nervous system, no history of systemic illness, and no history of alcoholism or drug dependency. Ten patients ( 8 men, 2 women; mean age, $37.8 \pm 9.3$ years; mean formal education, $14.5 \pm 1.9$ years) returned for follow-up visits $>9$ months after the baseline visit since this period is close to the end of a stage in which sustained spontaneous recovery of cognitive function is expected. ${ }^{23,24}$ Ten patients ( 8 men, 2 women; mean age, $32.9 \pm 11.6$ years; mean formal education, $15.8 \pm 1.7$ years) did not return for follow-up visits since of moving to a different state or unwillingness to participate. Patients underwent the same MR imaging protocol and clinical assessment at both baseline (mean interval after injury, $22.1 \pm 15.4$ days; range, 5-54 days) and follow-up visits (mean interval after baseline visit, $369.6 \pm 112.1$ days; range, 238-584 days). Sixteen healthy con- trols matched with patients according to sex, age, and formal education ( 13 men, 3 women; mean age, $35.1 \pm 11.9$ years; mean formal education, $16.6 \pm 1.9$ years) underwent the same MR imaging protocols and clinical assessment as patients at both baseline and follow-up visits in a single session. Approval for the study was obtained from the Institutional Review Board of the New York University School of Medicine, and all participants provided informed written consent.

\section{Clinical Assessment}

Clinical assessment was conducted on the same day that subjects underwent MR imaging and was administered by a psychologist blinded to imaging results. Postconcussive symptoms were assessed by using the Postconcussive Severity Scale. ${ }^{25}$ Cognitive impairment was assessed by using the neuropsychological battery discussed in the On-line Supplement, Section 1, which was designed to yield measures for attention, concentration, executive functioning, memory, learning, and information processing. Results from each test were converted to $z$ scores by using published norms, for easier comparison between subject groups, with higher indices indicative of better performance. Patients who scored at or below the fifth percentile ( -1.6 SDs below the normative mean) on $\geq 2$ tests compared with controls were categorized as cognitively impaired. ${ }^{26}$ Depression, anxiety, and fatigue were also assessed by using the Beck Anxiety Inventory, ${ }^{27}$ the Beck Depression Inventory, ${ }^{28}$ and the Fatigue Severity Scale, ${ }^{29}$ respectively. Quality of life was assessed by using the Satisfaction with Life Scale. $^{30}$

\section{Image Acquisition}

MR imaging was performed in all subjects by using a 3T Magnetom Tim Trio whole-body scanner (Siemens, Erlangen, Germany) with a 12-channel head matrix coil and the sequence protocol discussed in the On-line Supplement, Section 2. Images from all sequences were evaluated by a neuroradiologist and analyzed for structural abnormalities, including microinfarcts and gliotic spots in the region of the small penetrating arteries and arterioles.

\section{Data Processing and Analysis}

Data processing and analysis are discussed in the On-line Supplement, Section 3. Deep gray matter data analysis was performed by using ImageJ (National Institutes of Health, Bethesda, Maryland) software with manually drawn ROIs, and WM data analysis was performed by using the fMRI of the Brain Software Library (http://www.fmrib.ox.ac.uk/fsl) with the tract-based spatial statistics package. $^{31}$

\section{Statistical Analysis}

Statistical analysis is discussed in the On-line Supplement, Section 4. All $P$ values reported were 2 -sided, adjusted for numeric covariates, and declared statistically significant only with $P<.05$. While $P$ values reported were not adjusted for multiple comparisons, explicit mention has been made with regard to results that would have remained statistically significant after Bonferroni correction. 


\begin{tabular}{|c|c|c|c|c|c|c|c|c|}
\hline Patient & $\begin{array}{c}\text { Age at } \\
\text { Baseline } \\
\text { (yr)/Sex }\end{array}$ & $\begin{array}{l}\text { Cause of } \\
\text { Injury }\end{array}$ & $\begin{array}{l}\text { Days since } \\
\text { Injury at } \\
\text { Baseline }\end{array}$ & $\begin{array}{l}\text { GCS Score } \\
\text { at Baseline }\end{array}$ & $\begin{array}{l}\text { T2WI Findings } \\
\text { at Baseline }\end{array}$ & $\begin{array}{l}\text { Days since } \\
\text { Baseline at } \\
\text { Follow-Up }\end{array}$ & $\begin{array}{c}\text { GCS Score } \\
\text { at Follow-Up }\end{array}$ & $\begin{array}{l}\text { T2WI Findings } \\
\text { at Follow-Up }\end{array}$ \\
\hline 1 & $18 / \mathrm{M}$ & Assault & 10 & 15 & Normal & 238 & 15 & Normal \\
\hline 2 & $20 / M$ & Assault & 19 & 14 & Normal & & & \\
\hline 3 & $23 / \mathrm{M}$ & Assault & 18 & 15 & Normal & & & \\
\hline 4 & $25 / \mathrm{M}$ & Assault & 17 & 15 & Normal & & & \\
\hline 5 & $26 / F$ & Ped/auto & 14 & 15 & Normal & & & \\
\hline 6 & $27 / F$ & MVA & 15 & 15 & Normal & & & \\
\hline 7 & $28 / \mathrm{M}$ & Fall & 11 & 15 & Normal & & & \\
\hline 8 & $29 / M$ & Fall & 13 & 15 & Normal & 394 & 15 & Normal \\
\hline 9 & $32 / \mathrm{M}$ & Assault & 17 & 15 & Normal & 308 & 15 & Normal \\
\hline 10 & $33 / F$ & Fall & 44 & 15 & Normal & 413 & 15 & Normal \\
\hline 11 & $36 / M$ & Sports & 24 & 15 & Normal & & & \\
\hline 12 & $36 / M$ & Sports & 25 & 15 & Normal & 351 & 15 & Normal \\
\hline 13 & $39 / \mathrm{M}$ & Fall & 31 & 15 & Normal & & & \\
\hline 14 & $41 / \mathrm{M}$ & Fall & 3 & 15 & Normal & 505 & 15 & Normal \\
\hline 15 & $41 / \mathrm{M}$ & Fall & 5 & 15 & Normal & 274 & 15 & Normal \\
\hline 16 & $42 / \mathrm{M}$ & Fall & 5 & 14 & Normal & 384 & 15 & Normal \\
\hline 17 & $45 / F$ & Ped/auto & 55 & 15 & Normal & 584 & 15 & Normal \\
\hline 18 & $50 / \mathrm{M}$ & Fall & 54 & 15 & Normal & & & \\
\hline 19 & $51 / \mathrm{M}$ & MVA & 22 & 14 & $\begin{array}{l}\text { Left temporal lobe } \\
\text { encephalomalacia }\end{array}$ & 245 & 15 & $\begin{array}{l}\text { Left temporal lobe } \\
\text { encephalomalacia }\end{array}$ \\
\hline 20 & $57 / M$ & Assault & 40 & 15 & Normal & & & \\
\hline
\end{tabular}

Note:-GCS indicates Glasgow Coma Scale; MVA, motor vehicle accident; Ped/auto, pedestrian struck by an automobile.

\section{RESULTS}

Patient demographics and clinical data are summarized in the Table. Conventional MR imaging scans showed the presence of left temporal lobe encephalomalacia in 1 of the 20 patients at both baseline and follow-up visits, while no abnormalities were observed in any of the controls. Seventeen patients at baseline visit had an emergency department Glasgow Coma Scale score of 15, and 3 had a score of 14, while all 10 patients at follow-up visit had a score of 15. All patients had postconcussive symptoms such as headache, photophobia, nausea, dizziness, fatigue, memory deficits, and sleep disturbances except 7 patients at baseline visit and 6 patients at follow-up visit who reported a complete recovery at the time they were studied.

Clinical assessment results for the mean and SDs of neuropsychological battery $z$ scores obtained from the control and patient groups, as well as cases in which they demonstrated significant differences, are shown in the On-line Table. Ten of the 20 patients were deemed cognitively impaired at baseline visit (50\%), and 3 of these among the 10 patients who returned remained cognitively impaired at the follow-up visit (30\%). For patients at baseline visit, most cognitive impairment was found in the domains of executive functioning (25\%), memory and learning (30\%), and information processing (30\%); and for patients at follow-up visit, most cognitive impairment was found in the domains of memory and learning (30\%) and information processing (20\%). Compared with controls, patients at baseline visit also showed significantly higher scores in the Postconcussive Severity Scale $(P=.03)$ as well as the Beck Anxiety Inventory $(P=.03)$ and the Beck Depression Inventory $(P=.02)$, while patients at follow-up visit only showed significantly higher scores in the Beck Depression Inventory $(P=.04)$.

Examples of MK, FA, MD, and CBF maps are shown in Online Figs 1 and 2. Variations in measurement differed across deep gray matter ROIs for each DTI, DKI, and ASL measure with the coefficient of variation ranging from $2.3 \%-5.8 \%$ in $\mathrm{MK}, 3.5 \%-$ $10.2 \%$ in $\mathrm{FA}, 2.5 \%-8.4 \%$ in $\mathrm{MD}$, and $10.3 \%-20.4 \%$ in CBF.

Comparisons between controls and patients at both baseline visit and follow-up in terms of DTI, DKI, and ASL measures in the thalamus and total WM are shown in Figs 1 and 2. When patients at baseline visit were compared with controls, they showed significantly lower MK, FA, and CBF and higher MD in the thalamus (MK, FA, MD, and CBF: $P<.01$ ), the internal capsule (MK and FA: $P<.01$; MD: $P=.02$ ), the external capsule (MK and FA: $P<.01$ ), the corpus callosum (MK and MD: $P<$ .01 FA: $P=.01$ ), the cingulum (MK, FA, and MD: $P<.01$ ), the optic radiations (MK, FA, and MD: $P<.01$ ), the centrum semiovale (MK and FA: $P<.01$; $\mathrm{MD}: P=.02$ ), total deep gray matter (MK and FA: $P<.01$; MD: $P=.01$ ), and total WM (MK, FA, and MD: $P<.01)$. All differences remained statistically significant after Bonferroni correction. Patients at baseline visit also showed significant associations among DTI, DKI, and ASL measures in the thalamus (MK and CBF: $r=0.74, P<.01$; FA and CBF: $r=0.71, P<.01)$ and among DTI, DKI, and ASL measures in the thalamus and total WM (MD thalamus and MK total WM: $r=-0.56, P=.01$; MD thalamus and MD total WM: $r=0.67$, $P<.01$; CBF thalamus and MK total WM: $r=0.55, P=.01$ ).

When patients at follow-up visits were compared with controls, they showed significantly lower MK and CBF and higher MD in the thalamus (MK and CBF: $P<.01$ ), the corpus callosum (MK: $P<.01$ ), the cingulum (MK: $P<.01)$, the optic radiations (MK: $P=.02)$, the centrum semiovale (MK: $P<.01)$, total deep gray matter (MK: $P=.03)$, and total WM (MK: $P<.01$; MD: $P=$ $.03)$. All differences remained statistically significant after Bonferroni correction except for MK in total deep gray matter and MD in total WM. Patients at follow-up visit showed no significant associations among DTI, DKI, and ASL measures in the thalamus or DTI, DKI, and ASL measures in the thalamus and total WM.

When results of patients at follow-up visit were compared with

AJNR Am J Neuroradiol 34:951-57 May 2013 www.ajnr.org 
those at their baseline visit, they showed significantly higher FA and lower MD in the thalamus (FA and MD: $P<.01$ ), the internal capsule (MD: $P=.02$ ), and total deep gray matter $(\mathrm{FA}: P<.01)$ and higher $\mathrm{MD}$ in the corpus callosum $(P<.01)$ and total $\mathrm{WM}(P<.01)$. All differences remained statistically significant after Bonferroni correction.

When patients who were cognitively impaired were compared with those cognitively unimpaired at the baseline visit, they showed significantly lower MK in the thalamus $(P<.01)$ and FA in the optic radiations $(P=.02)$, which is shown in Fig 3. Only the difference for MK in the thalamus remained significant after Bonferroni correction. In addition, patients at baseline visit showed significant associations between DTI and DKI regional measures and neuropsychological $z$ score results (MK thalamus and Digit Span Test: $r=0.62, P=.01$; MK thalamus and Symbol Digit Modality Test: $r=0.66, P=.01 ; \mathrm{MD}$ external capsule and California Learning Verbal Test Total Recall: $r=0.57, P=.03$; MD corpus callosum and California Learning Verbal Test Total

Recall: $r=0.54, P=.04$; MK cingulum and Rey Complex Figure Test Short-Term Recall: $r=-0.57, P=.03$; MK centrum semiovale and Rey Complex Figure Test Short-Term Recall: $r=-0.60, P=.02$; MK total WM and Rey Complex Figure Test Short-Term Recall: $r=$ $-0.54, P=.04$; MD total WM and California Learning Verbal Test Total Recall: $r=0.57, P=.04)$.

When cognitively impaired patients were compared with cognitively unimpaired patients at follow-up visit, they showed no significant differences in DTI, DKI, and ASL regional measures. In addition, patients at follow-up visit showed no significant associations among DTI, DKI, and ASL regional measures and neuropsychological $z$ score results.

\section{DISCUSSION}

This study shows that the combined use of DTI, DKI, and ASL might improve the identification and characterization of regional brain injury in patients with MTBI cross-sectionally and possibly also longitudinally. The information provided might be useful in understanding pathophysiology in MTBI and cognitive impairment at baseline, though the small number of patients examined did not predict outcome after a short follow-up period.

In agreement with previous DTI investigations of MTBI, patients in comparison with controls showed lower FA and higher MD in various WM regions measured. ${ }^{2,7,9,10,16,17}$ More interesting, patients also showed lower MK and CBF in the thalamus and these regions as well as dynamic changes during a short follow-up period. Each of these factors has been sparsely investigated. ${ }^{3}$

An exact interpretation of DTI, DKI, and ASL measures with respect to the complex cellular architecture of neural tissue is still under investigation and is not yet completely understood. In the
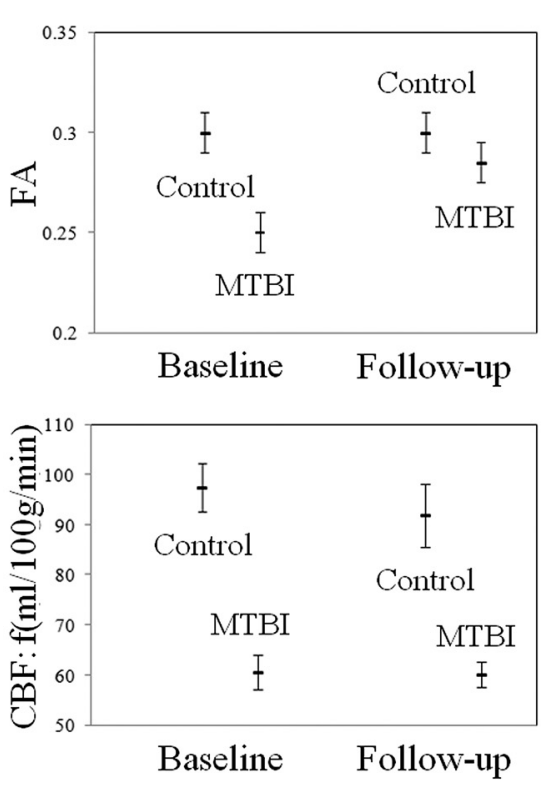

\section{Thalamus}

G 1. Plots displaying age- and sex-adjusted means (hash marks) and standard errors (lines) for patients with $\mathrm{MTBI}$ at follow-up visit. When patien, patients with $\mathrm{MTBl}$ at baseline visit, and they show significantly lower MK, FA, and CBF and higher MD at baseline visit (MK, FA, MD, and CBF: $P<.01$ ) and significantly lower MK and CBF at follow-up visit (MK and CBF: $P<.01$ ). All differences remain statistically significant after Bonferroni correction.

case of DTI, results from experimental animal models suggest that a decrease in FA and an increase in MD may be related to altered WM microstructural integrity and loss of axonal membranes and myelin sheaths. ${ }^{32}$ In the case of DKI, a decrease in MK has been inferred to suggest a diminution in overall diffusional heterogeneity associated with degenerative processes leading to neuronal shrinkage $e^{18-20,33}$ and changes in axonal and myelin density. ${ }^{34} \mathrm{Re}-$ sults from a recent study using DKI to investigate an experimental animal model of MTBI also found, from immunohistochemistry analysis of brain tissue sections, that MK might be sensitive to changes associated with reactive astrogliosis. ${ }^{35}$ In the case of ASL, results from experimental animal models and analytic models suggest that a decrease in $\mathrm{CBF}$ is related to a reduction in blood delivered to the vascular bed. ${ }^{36}$

There is some evidence to suggest that the thalamus might play a role in MTBI as a result of impairment, ${ }^{4-15}$ but very few studies have explored the susceptibility of this region to structural and functional damage during such injury. This raises an important consideration as to whether thalamic damage in MTBI is the result of primary injury caused by immediate direct traumatic insult, secondary injury caused by long-term indirect degenerative processes related to WM, or both of these mechanisms, which do not necessarily have to be mutually exclusive. Among the investigations that have been conducted, Zhang et al $^{15}$ examined primary injury in MTBI by using a computational model to test the vulnerability of different human brain tissues and found that the highest shear stress levels were localized to the thalamus and midbrain. In theory, this could result in not only structural damage to thalamic gray matter nuclei and WM bundles but also in perfusion decreases caused by either similar distress to capillaries asso- 

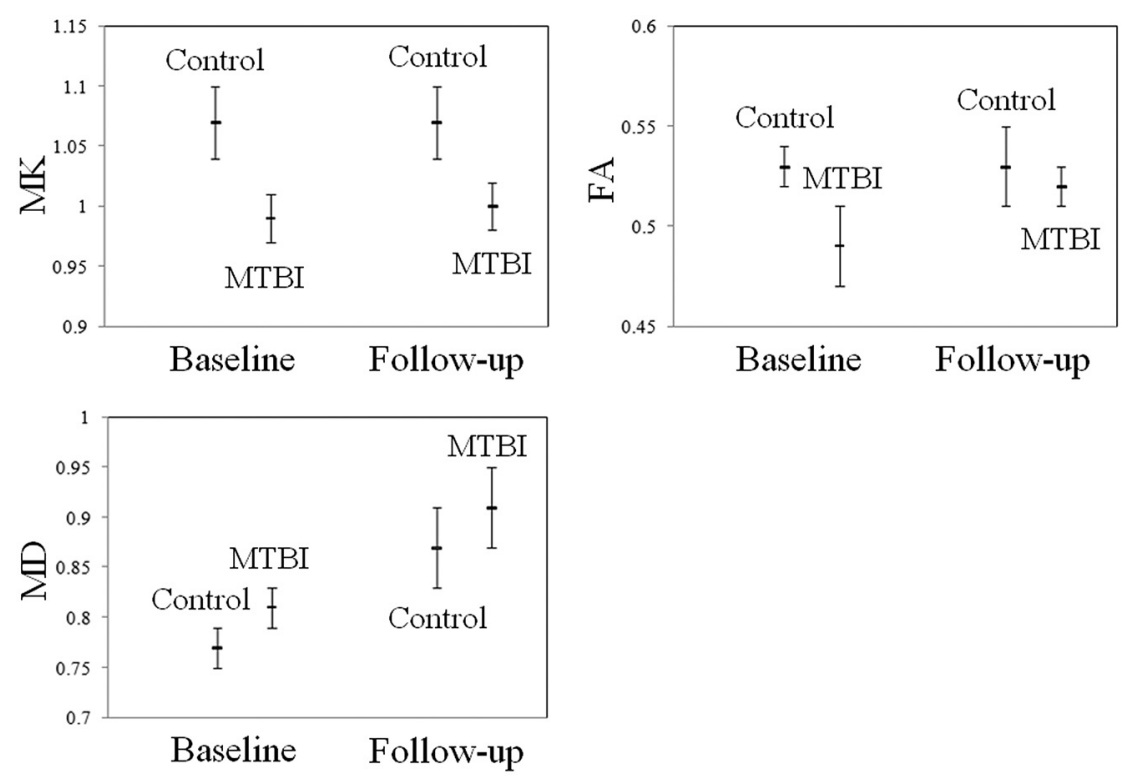

\section{Total white matter}

FIG 2. Plots displaying age- and sex-adjusted means (hash marks) and standard errors (lines) for MK, FA, and MD in the total WM of controls, patients with MTBI at baseline visit, and patients with MTBI at follow-up visit. When patients with MTBI are compared with controls, they show significantly lower MK and FA and higher MD at baseline visit (MK, FA, and MD: $P<.01$ ) and significantly lower MK at follow-up visit $(P<.01)$. All differences remain statistically significant after Bonferroni correction.

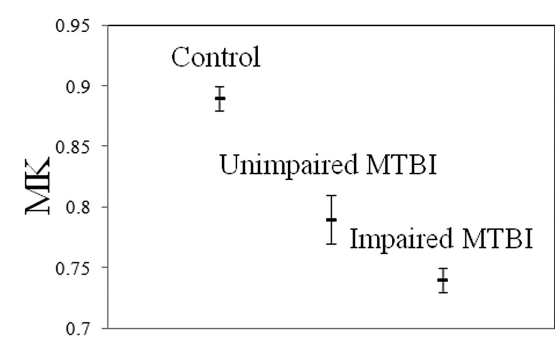

Thalamus

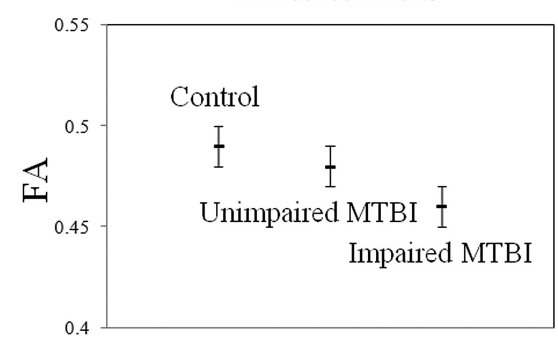

Optic radiations

FIG 3. Plots displaying age, sex, and time since injury adjusted means (hash marks) and standard errors (lines) for MK in the thalamus and FA in the optic radiations of controls and cognitively unimpaired patients with MTBI and cognitively impaired patients with MTBI at baseline visit. When cognitively impaired patients with MTBI are compared with cognitively unimpaired patients with MTBI, they show significantly lower MK in the thalamus $(P<.01)$ and FA in the optic radiations $(P=.04)$. Only the difference for $M K$ in the thalamus remains significant after Bonferroni correction.

ciated with the thalamoperforating arteries, for example, or a reduction in the need for oxygen and nutrients supplied by these capillaries, because neurons have become functionally impaired or have undergone apoptosis. In contrast, Anderson et $\mathrm{al}^{5}$ examined secondary injury in MTBI by using an experimental human model and found that if a lesion interrupts WM fibers connecting the cerebral cortex and the thalamus, then target cells in both regions might undergo apoptosis. As a corollary to this, it is possible that such damage could also lead to a loss of control by the cerebral cortex over perfusion and permeability of blood vessels supplying the thalamus, helping to contribute another source of secondary injury in MTBI. ${ }^{37}$ In the current study, the association in patients among DTI, DKI, and ASL measures in the thalamus and total WM suggests the presence of secondary injury in the thalamus in MTBI. Since, however, these were only moderate associations and there were also associations observed in patients among DTI, DKI, and ASL measures in the thalamus, the results also suggest that, at least in part, primary injury also occurs in the thalamus in MTBI.

Longitudinal data presented in this study showed that patients exhibited variable changes in different brain regions for DTI, DKI, and ASL measures with time. In some brain regions, imaging measures improved with time, suggesting that tissue repair had occurred; and in other brain regions, imaging measures only partially recovered with time and remained significantly impaired compared with controls, suggesting that incomplete tissue repair had occurred; and in other brain regions, imaging measures worsened with time, suggesting that further tissue damage and degeneration had occurred.

These results are consistent with and add to findings from previous DTI longitudinal investigations ${ }^{16,38}$ and DKI and ASL cross-sectional investigations ${ }^{6,7}$ of MTBI, which have been few in number. ${ }^{3}$ In the case of the thalamus, previous cross-sectional investigations reported that compared with controls, patients showed decreased MK, FA, and CBF and increased MD immediately following injury, which was partially or completely corrected several weeks to months later. ${ }^{6,7}$ In the current study, it was found in the thalamus that when patients at follow-up visit were compared with their baseline visit, 10\% showed lower MK and $50 \%$ showed lower CBF. The results in MK might suggest the presence of degenerative processes in some patients associated with neuronal shrinkage and changes in axonal and myelin density and inflammation marked by reactive astrogliosis. This understanding could be supported in part by the results in CBF since such degenerative processes generally occur along with hypoxia, which can be either a cause or effect of neuronal weakening and destruction. In the case of WM, previous longitudinal investigations reported that compared with controls, patients showed decreased FA in a number of white matter regions immediately following injury, which were partially or completely corrected 
several weeks to months later. ${ }^{16,38}$ In the current study, it was found that in total WM, when findings of patients at follow-up visit were compared with those at their baseline visit, 33\% showed lower MK and FA and 80\% showed increased MD. In addition to the possible meaning of the results in MK previously discussed, the results in FA and MD might suggest the presence of Wallerian degeneration in most patients, associated with disruption, disorganization, and loss of axonal membranes and myelin sheaths.

The most important finding in this study is that when cognitively impaired patients were compared with cognitively unimpaired patients at baseline visit, they showed significantly lower $\mathrm{MK}$ and FA in the thalamus and optic radiations. In addition, patients at baseline visit showed associations between MK in the thalamus and neuropsychological performance on tests of attention, concentration, and information processing as well as associations between $\mathrm{MK}$ and $\mathrm{MD}$ in various WM regions measured and neuropsychological performance on tests of attention, concentration, memory, learning, and information processing. Since these results were observed in patients only at baseline visit and not at follow-up visit, they might be viewed as suggesting that repair had occurred and was consistent with the longitudinal changes observed in DTI and DKI for various brain regions measured. Since, however, only $50 \%$ of patients at baseline exhibited cognitive impairment, caution should be exercised in interpreting these results because it is possible that some patients deemed cognitively unimpaired may also have undetected structural and/or functional damage that had not yet become apparent or show associations by using the methods of this study. Nevertheless, it appears that neuropsychological performance in MTBI might be complex and involve global interaction among many brain regions. This finding is consistent with previous studies of MTBI which have also reported that the integrity of WM pathways in the corpus callosum, cingulum, and frontal and temporal lobes is associated with performance on neuropsychological tests of attention, concentration, memory, learning, and information processing. ${ }^{3}$

Unlike WM, however, the thalamus has not been widely accepted as a probable anatomic correlate for cognitive impairment in MTBI and therefore remains largely uninvestigated with regard to this condition. ${ }^{39}$ Despite the lack of research, there is evidence that the thalamus might contribute to cognitive impairment in MTBI. As the principal relay station for the brain, the thalamus has influence over many global activities through reciprocal projections to the entire cerebral cortex and, if damaged, could produce much of the clinical nonfocalized sequelae observed in MTBI. ${ }^{40}$ A number of investigations have reported associations between the thalamus and neuropsychological performance in domains such as attention, concentration, and information processing, which are relevant to this study, for a variety of neurologic diseases including, preliminarily, MTBI. ${ }^{6,7,9,10}$

Since it was found that in the thalamus and most WM regions measured during the time of this study, compared with controls, patients exhibited significantly lower MK and CBF, and tended to normalize FA and MD, it is possible that DKI and ASL might be detecting aspects of injury for which DTI lacks sensitivity. For example, if neuronal recovery or degeneration is occurring, then $\mathrm{MK}$ and perhaps also CBF might reflect the underlying dynamic complexities of these processes such as subtle precursory changes in axonal and myelin density or the presence of inflammation marked by reactive astrogliosis, while FA and MD might reflect either the beneficial or detrimental results of the ongoing activity of these processes. In addition, CBF might reflect changes in vascular and cellular function associated with neuronal recovery or degeneration for which MK, FA, and MD lack sensitivity. The ability to identify initial changes in axonal and myelin density, the presence of reactive astrogliosis, or changes in vascular and cellular function could be extremely useful since they have the potential to be sensitive markers for diseased tissue and could play a role in predicting clinical outcome and could help facilitate early medical and rehabilitative interventions that might reduce long-term or permanent deficits. ${ }^{2}$

A number of potential limitations can be found with this study. One issue is the relatively small number of patients examined at baseline visit and the fact that only half returned for follow-up visits, which detract statistical power from the conclusions that can be drawn from the results, including the potential prognostic value of the techniques used. Another issue is that more extensive pre-examination and post-examination intervals as well as longer-term follow-up visits are necessary to better track the development of damage and recovery. These issues will be addressed in subsequent studies.

\section{CONCLUSIONS}

It has been demonstrated that through the combined use of DTI, DKI, and ASL, it is feasible to detect subtle brain tissue injury in both the thalamus and WM of patients with MTBI at both baseline and follow-up visits in a longitudinal study. Since patients exhibited significant differences from controls for $\mathrm{MK}$ and $\mathrm{CBF}$ in the thalamus and for $\mathrm{MK}, \mathrm{FA}$, and $\mathrm{MD}$ in various $\mathrm{WM}$ regions measured at both baseline and follow-up visits and since these measures were also associated with neuropsychological performance at baseline, DTI, DKI, and ASL might be useful for investigating dynamic changes and cognitive impairment during a short follow-up period, though it was not possible to predict outcome with the small cohort examined in this study.

\section{ACKNOWLEDGMENTS}

We thank the following individuals from the New York University School of Medicine: Laura Miles for the neuropsychological assessment of subjects; Kelly A. McGorty for technical support; Joseph Reaume for coordinating subjects; and Henry Rusinek and Edward Ziff for advice on the study.

\section{REFERENCES}

1. National Center for Injury Prevention and Control. Report to Congress on Mild Traumatic Brain Injury in the United States: Steps to Prevent a Serious Public Health Problem. Atlanta: Centers for Disease Control and Injury Prevention; 2003

2. Miles L, Grossman RI, Johnson G, et al. Short-term DTI predictors of cognitive dysfunction in mild traumatic brain injury. Brain Inj 2008;22:115-22

3. Grossman EJ, Inglese M, Bammer R. Mild traumatic brain injury: is diffusion imaging ready for primetime in forensic medicine? Top Magn Reson Imaging 2010;21:379-86

4. Abdel-Dayem HM, Abu-Judeh H, Kumar M, et al. SPECT brain per- 
fusion abnormalities in mild or moderate traumatic brain injury. Clin Nucl Med 1998;23:309-17

5. Anderson CV, Wood DM, Bigler ED, et al. Lesion volume, injury severity, and thalamic integrity following head injury. J Neurotrauma 1996;13:59-65

6. Ge Y, Patel MB, Chen Q, et al. Assessment of thalamic perfusion in patients with mild traumatic brain injury by true FISP arterial spin labelling MR imaging at 3T. Brain Inj 2009;23:666-74

7. Grossman EJ, Ge Y, Jensen JH, et al. Thalamus and cognitive impairment in mild traumatic brain injury: a diffusional kurtosis imaging study. J Neurotrauma 2012;29:2318-27

8. Henninger N, Sicard KM, Li Z, et al. Differential recovery of behavioral status and brain function assessed with functional magnetic resonance imaging after mild traumatic brain injury in the rat. Crit Care Med 2007;35:2607-14

9. Little DM, Kraus MF, Joseph J, et al. Thalamic integrity underlies executive dysfunction in traumatic brain injury. Neurology 2010;74: 558-64

10. Messé A, Caplain S, Paradot G, et al. Diffusion tensor imaging and white matter lesions at the subacute stage in mild traumatic brain injury with persistent neurobehavioral impairment. Hum Brain Mapp 2011;32:999-1011

11. Wood DM, Bigler ED. Diencephalic changes in traumatic brain injury: relationship to sensory perceptual function. Brain Res Bull 1995;38:545-49

12. Jacobs A, Put E, Ingels M, et al. One-year follow-up of technetium99m-HMPAO SPECT in mild head injury. J Nucl Med 1996;37: 1605-09

13. Nakayama N, Okumura A, Shinoda J, et al. Relationship between regional cerebral metabolism and consciousness disturbance in traumatic diffuse brain injury without large focal lesions: an FDGPET study with statistical parametric mapping analysis. J Neurol Neurosurg Psychiatry 2006;77:856-62

14. Stamatakis EA, Wilson JT, Hadley DM, et al. SPECT imaging in head injury interpreted with statistical parametric mapping. J Nucl Med 2002;43:476-83

15. Zhang L, Yang KH, King AI. A proposed injury threshold for mild traumatic brain injury. J Biomech Eng 2004;126:226-36

16. Arfanakis K, Haughton VM, Carew JD, et al. Diffusion tensor MR imaging in diffuse axonal injury. AJNR Am J Neuroradiol 2002;23: $794-802$

17. Inglese M, Makani S, Johnson G, et al. Diffuse axonal injury in mild traumatic brain injury: a diffusion tensor imaging study. J Neurosurg 2005;103:298-303

18. Jensen JH, Helpern JA. MRI quantification of non-Gaussian water diffusion by kurtosis analysis. NMR Biomed 2010;23:698-710

19. Jensen JH, Helpern JA, Ramani A, et al. Diffusional kurtosis imaging: the quantification of non-gaussian water diffusion by means of magnetic resonance imaging. Magn Reson Med 2005;53: $1432-40$

20. Lu H, Jensen JH, Ramani A, et al. Three-dimensional characterization of non-gaussian water diffusion in humans using diffusion kurtosis imaging. NMR Biomed 2006;19:236-47

21. Grossman EJ, Zhang K, An J, et al. Measurement of deep gray matter perfusion using a segmented true-fast imaging with steady-state precession (true-FISP) arterial spin-labeling (ASL) method at 3T. J Magn Reson Imaging 2009;29:1425-31

22. Esselman PC, Uomoto JM. Classification of the spectrum of mild traumatic brain injury. Brain Inj 1995;9:417-24

23. Cancelliere C, Cassidy JD, Cote P, et al. Protocol for a systematic review of prognosis after mild traumatic brain injury: an update of the WHO Collaborating Centre Task Force findings. Syst Rev 2012;1:17

24. Szymanski HV, Linn R. A review of the postconcussion syndrome. Int J Psychiatry Med 1992;22:357-75

25. Aubry M, Cantu R, Dvorak J, et al. Summary and agreement statement of the First International Conference on Concussion in Sport, Vienna 2001: recommendations for the improvement of safety and health of athletes who may suffer concussive injuries. Br J Sports Med 2002;36:6-10

26. Lezak MD, Howieson DB, Loring DW. Neuropsychological Assessment. 4th ed. New York: Oxford University Press; 2004

27. Beck AT, Epstein N, Brown G, et al. An inventory for measuring clinical anxiety: psychometric properties. J Consult Clin Psychol 1988;56:893-97

28. Beck AT, Ward CH, Mendelson M, et al. An inventory for measuring depression. Arch Gen Psychiatry 1961;4:561-71

29. Krupp LB, LaRocca NG, Muir-Nash J, et al. The Fatigue Severity Scale. Application to patients with multiple sclerosis and systemic lupus erythematosus. Arch Neurol 1989;46:1121-23

30. Diener E, Emmons RA, Larsen RJ, et al. The Satisfaction with Life Scale. J Pers Assess 1985;49:71-75

31. Smith SM, Jenkinson M, Johansen-Berg H, et al. Tract-based spatial statistics: voxelwise analysis of multi-subject diffusion data. Neuroimage 2006;31:1487-505

32. Basser PJ. Inferring microstructural features and the physiological state of tissues from diffusion-weighted images. NMR Biomed 1995; 8:333-44

33. Wu EX, Cheung MM. MR diffusion kurtosis imaging for neural tissue characterization. NMR Biomed 2010;23:836-48

34. Fieremans E, Jensen JH, Helpern JA. White matter characterization with diffusional kurtosis imaging. Neuroimage 2011;58:177-88

35. Zhuo J, Xu S, Proctor JL, et al. Diffusion kurtosis as an in vivo imaging marker for reactive astrogliosis in traumatic brain injury. Neuroimage 2012;59:467-77

36. Buxton RB. Quantifying CBF with arterial spin labeling. J Magn Reson Imaging 2005;22:723-26

37. Feig SL, Guillery RW. Corticothalamic axons contact blood vessels as well as nerve cells in the thalamus. Eur J Neurosci 2000;12:2195-98

38. Mayer AR, Ling J, Mannell MV, et al. A prospective diffusion tensor imaging study in mild traumatic brain injury. Neurology 2010;74: $643-50$

39. Mittl RL, Grossman RI, Hiehle JF, et al. Prevalence of MR evidence of diffuse axonal injury in patients with mild head injury and normal head CT findings. AJNR Am J Neuroradiol 1994;15:1583-89

40. Sherman SM, Guillery RW. Exploring the Thalamus and Its Role in Cortical Function. 2nd ed. Cambridge, Massachusetts: The MIT Press; 2006 